\title{
The Preparation of Solid Polymer Microspheres Immune Adsorbent and Its Adsorption Performance of Aflatoxin M1
}

\author{
Zhang xiaozhou ${ }^{1, a^{*}}$, Pei shichun ${ }^{2, b}$ and Liang meng ${ }^{1, c}$ \\ ${ }^{1}$ College of Materials Science and Engineering, Qiqihar University, Qiqihar 161006, China \\ ${ }^{2}$ College of Food and Biological Engineering, Qiqihar University, Qiqihar 161006, China

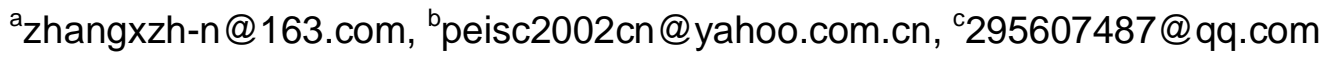 \\ * please mark the corresponding author with an asterisk
}

Keywords: Copolymer Microspheres; Aflatoxin M1; Immune adsorbent; Adsorption Abstract. In this paper, the immune adsorbents were prepared for adsorption of aflatoxin M1 in milk. The copolymerization of glyceryl methacrylate (GMA) and methyl methacrylate (MMA) microspheres were prepared using dispersion polymerization with azodiisobutyronitrile (AIBN) as initiator in ethanol-aqueous medium in the presence of polyvinylpyrrolidone (PVP) as dispersant under various conditions. Using epoxy groups on the microsphere surface AFM1 monoclonal antibody were coupled on the microspheres .The adsorption quantity of monoclonal antibodies for aflatoxin M1 in the milk was more than $1.2 \mu \mathrm{g} / \mathrm{g}$. The results showed that the selective adsorption for aflatoxin M1 in milk of immune adsorbent is good. It was an ideal immune adsorption material for removed of aflatoxin M1 in milk.

\section{Introduction}

Aflatoxin M1 (Aflatoxin M1, AFM1) because of its mainly exists in milk, so the original is also called the "poison" milk, is discovered by De Iongh etc. [1] after published in 1964 in the journal Nature, with the deepening of the research is found AFM1 Aflatoxin B1 (AFB1, Aflatoxin B1) by the series of cytochrome P450 enzymes in the body under the action of the hydroxylation, formed by hydroxyl of AFB1 metabolites is generally recognized as toxins detoxification form, however AFM1 cannot be viewed as an outgrowth of AFB1 detoxification, there are a number of studies have shown that AFM1 cellular toxicity and carcinogenicity, affect the dairy products security is one of the important pests of [2].

Due to aspergillus flavus are widespread in nature, and the growth condition of request is not high, grain and oil crops and feed is the most easy to breed, mold AFB1 often cause pollution, and then through the food chain transfer, so the indirect control milk AFM1 method is mainly through the strict management of feed intake lower mammals AFB1. But even if is to strengthen management and used the subsidy measures such as prevention is also difficult to completely eliminate the feed of AFB1, environment pollution, often AFB1 pollution events and related report ${ }^{[3]}$, AFB1 can pass through the feed intake and water transferred to the mammalian body, intake of AFB1 in mammals into AFM1 in vivo and is discharged through breast milk, causing the pollution of AFM1 of dairy ${ }^{[4]}$, as a result of fresh milk in before eating and processing process is not easy to directly use the methods such as chemical reagent, as a result, using highly specific monoclonal antibody and special carrier material preparation high specificity in vitro immune adsorbent, by AFM1 immune adsorption removal of milk residue is a one of the methods can be considered.

And as biological immobilized carrier, polymer microsphere has good mechanical strength, large specific surface area, good monodispersity and stability, and the surface of the microspheres can design the hydroxyl ${ }^{[5]}$, carboxyl ${ }^{[6]}$, aldehyde group ${ }^{[7]}$, epoxy group ${ }^{[8]}$ s such as a variety of functional group, can be used to react with biological molecules to achieve chemically bonded ${ }^{[9]}$. Methyl Glycidyl ester of acrylic (Glycidyl methacrylate, GMA) molecules contain lively epoxy group, with other monomer copolymerization preparation of polymer microsphere material.This experiment was prepared by dispersion polymerization method containing epoxy groups in the surface of the monodisperse Styrene/methyl glycidyl ester of acrylic acid copolymer (Poly real (Styrene - glycidyl methacrylate), P (St - GMA)) microspheres, use it as the carrier, will resist AFM1 monoclonal 
antibody coupling to the polymer microspheres for solid phase immune adsorbent, and the adsorption of milk AFM1 performance are analyzed.

\section{materials and methods}

\section{The instrument and equipment}

VICTOR X4 multifunctional enzyme mark, 96 hole microplate Wahser400, NanoVue 100 trace ultraviolet spectrophotometer (GE) in the United States;Ultrafiltration cup (Michigan manage Po company);S - 4300 scanning electron microscope (Japan's Hitachi);TDL - 5 large capacity at low speed centrifuge (changzhou jie experiment instrument manufacturing co., LTD.);TENSOR type 27 Fourier infrared spectrometer Brucker spectral instruments (Germany).

\section{Method}

\section{Preparation of poly (St - GMA) microspheres}

To a certain quality of polyvinyl pyrrolidone (Polyvinylpyrrolidone, PVP), add a certain mass ratio of ethanol and water form a dispersed system. And then adding dispersed system with airway, condenser pipe, the three in the bottle neck of the thermometer, ventilation with nitrogen at the same time, in the magnetic heating stirrer scattered $30 \mathrm{~min}$. First and then will dissolve azodiisobutyronitrile Styrene (Styrene, St) join, $30 \sim 40$ min will again after a certain mass ratio of methyl glycidyl ester of acrylic drip into the reaction system, St: GMA ratio range of $0.6 \sim 0.6$ (mass ratio). $12 \mathrm{~h}$ at $70{ }^{\circ} \mathrm{C}$, under a certain speed response, get bright white emulsion. With ethanol and water washing sediment above 3 times, respectively, the sediment in $30 \sim 40{ }^{\circ} \mathrm{C}$ under the condition of vacuum drying, for a quick product copolymer microspheres.

\section{Poly (St - GMA) microspheres in the determination of content of epoxy group}

Using hydrochloric acid-dioxane method determination of residual concentrations of epoxide group. The method USES the reaction between hydrochloric acid and epoxy group, chlorohydrin reaction of acid consumption at the same time. Dissolve a certain quality of polymer microspheres in 1, 4 -dioxane, add excessive concentration of $0.1 \mathrm{~mol} / \mathrm{L}$ hydrochloride, in $30{ }^{\circ} \mathrm{C}$ under the condition of stirring reaction $3 \mathrm{~h}$. Unreacted hydrochloric acid concentration in $0.1 \mathrm{~mol} / \mathrm{L}$ sodium hydroxide solution, titration with phenolphthalein as indicator. At least 5 times repeated measurement on each samples.

\section{Carrier coupling with antibodies}

To contain a certain amount of poly (St - GMA) microspheres in the conical flask batch to join with PBS as 1 (including $\mathrm{g} / \mathrm{mL}$ AFM1 monoclonal antibody solution resistance, conical flask in $4{ }^{\circ} \mathrm{C}$ oscillation reaction in the shaking table, in the process of the reaction by enzyme-linked immunoassay was developed for the determination of residual in reaction liquid mass fraction of AFM1 monoclonal antibody, when there were no changes in antibody mass fraction inference for microspheres fully complete coupling reaction with antibody. Determination

\section{Dynamic adsorption}

Add artificial AFM1 milk, equipped with immune adsorbent adsorption column, peristaltic pump with a silica gel catheter connected in sequence, and open cycle peristaltic pump. Milk add AFM1 after the mass concentration of 1,5 , including $20 \mathrm{~g} / \mathrm{L}$, adsorbent, each $0.3 \mathrm{~g}$ each milk for $30 \mathrm{~mL}$, total volume flow rate of $2 \mathrm{~mL} / \mathrm{min}$, the complete an adsorption cycle for $15 \mathrm{~min}$, and $0,15,30 \mathrm{~min}$ when collecting 200 (including L samples are measured respectively.

\section{enzyme-linked immunosorbent assay AFM1}

Enzyme-linked immunosorbent assay (Enzyme - linked immune sorbent assay, ELISA) reference puffy, such as taking AFM1 - BSA with PBS diluted by liquid package by Enzyme label plate, closed with 5\% skimmed milk powder solution, each hole plus 50 (including L AFM1 monoclonal antibody, then add dairy products (including $50 \mathrm{~L}$ under test, after a warm bath with horseradish peroxidase labeled mouse IgG antibody and sheep.opd color, after joining terminated liquid adsorption rate OD value measurement and calculation.Press type calculation adsorbent for AFM1 adsorption rate. 


\section{The results and analysis}

\section{The microstructure of the copolymer microspheres}

With trace sampler, anhydrous ethanol diluted sample form suspension oscillation, and then ultrasonic about $20 \mathrm{~min}$. Take a trickle in the sample plate of conductive scraps of paper, placed in a petri dish at room temperature, dry, with ion sputtering of the sample surface after spray gold, using SEM scanning directly (SEM test acceleration voltage is $20.0 \mathrm{kV}$ ), to observe the morphology of microspheres apparent.
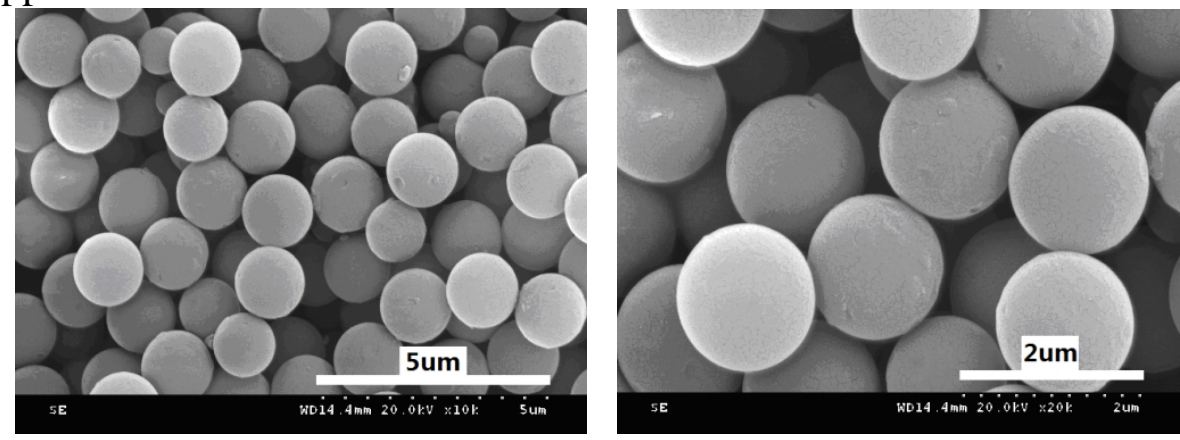

Fig.1 SEM images of P(St-GMA)composite particles

Microstructure by copolymer microspheres (figure 1) can be obtained, through adjusting experimental conditions, the dispersion polymerization can successfully controlled particle size, narrow particle size distribution was uniform copolymer ( $\mathrm{St}$ - GMA) microspheres, the particle size of the microspheres is about 1.7 microns, spherical rules, good monodispersity.

\section{A dynamic immune adsorption efficiency}

Based on the static adsorption experiment, immune to adsorbent for dynamic adsorption efficiency of the experimental results show that the preparation of specific immune adsorbent AFM1 adsorption rate in the milk can be adsorbed by more than $80 \%$ in one cycle of AFM1, high adsorption efficiency, basic adsorption reaction, can be completed within 15 min after the adsorption rate is no obvious change, maintain a relatively stable state (figure 5).

Dynamic adsorption experiments, for immune adsorption experiment of adsorbent mass is $0.3 \mathrm{~g}$, the volume of milk for $30 \mathrm{~mL}$, add milk solution has 5, 10 and 20 (including g/L AFM1, after cyclic dynamic adsorption, adsorption performance is shown in figure 3, add 5, 10 (including $\mathrm{g} / \mathrm{L}$, and in the cow's milk (including $20 \mathrm{~g} / \mathrm{L}$ AFM1 adsorption rate is $86 \%, 92 \%$ and $61 \%$, respectively, by calculation show that when the concentration of quality milk in more than 20 (including g/L AFM1 experiments with adsorbent adsorption $(0.3 \mathrm{~g})$ has been in a saturated state, adsorption, unable to continue to AFM1 for (including $10 \mathrm{~g} / \mathrm{L}$ in the milk the adsorption rate was $92 \%$, as a result, preparation of immune adsorbent adsorption AFM1 can preliminary judgment ability above (including $1.2 \mathrm{~g} / \mathrm{g}$, has high adsorption performance, is expected to be used in the dairy adsorption to remove AFM1 technology provides a new material.

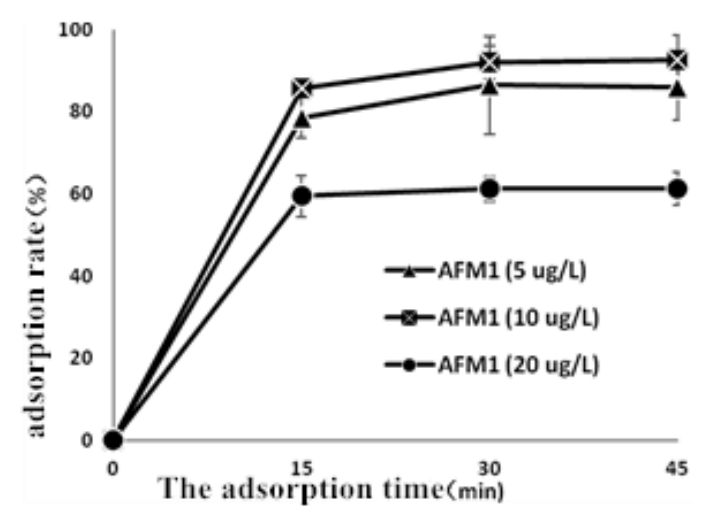

Fig.2 The absorption efficiency for AFM1 of the immunoadsorbents in milk 


\section{conclusion}

By using the method of dispersion polymerization of the synthesis of monodisperse poly ( $\mathrm{St}$ GMA) microspheres, the epoxy groups on the surface of the microspheres can AFM1 monoclonal antibody response, and the antibody immobilized on the surface of microspheres, get specific immune adsorbent.Immune adsorbent in the milk of AFM1 good adsorption effect, using monoclonal antibody immune adsorption technology has potential application prospect in the field of food safety.

\section{Acknowledgment:}

Supported by Natural Science Foundation of Heilongjiang Province(C201225) and project grant fr om Heilongjiang provincial department of education(Grand No12541856)

\section{References}

[1] Xiao-lian zhao ,dong-sheng zhang, Chun -cheng Zhao, etc. The harm of aflatoxin M1, pollution status quo and progress test method [J]. Chinese journal of health inspection,, 2004, 14(3): 266-269.

[2] Creppy E E. Update of survey, regulation and toxic effects of mycotoxins in Europe[J]. Toxicology Letters, 2002, 127(1/3): 19-28.

[3] Firmin S, Morgavi D P, Yiannikouris A, et al. Effectiveness of modified yeast cell wall extracts to reduce aflatoxin B1 absorption in dairy ewes[J]. Journal of Dairy Science, 2011, 94(11): 5611-5619.

[4] Rong-rong Zhang, Zi-li Xu, Shu-xin Li Rich in surface hydroxyl of monodisperse PS microsphere preparation and characterization of $[\mathrm{J}]$. Journal of materials science and technology, 2010, 18(5): 706-711.

[5] Zeravik J, Skryjova K, Nevorankova Z, et al. Development of direct ELISA for the determination of 4-nonylphenol and octylphenol[J]. Analytical Chemistry, 2004, 76 (4): 1021-1027.

[6] Xiao Xiao, Yang Xu, Liu Ting, et al. Preparing a highly specific inert immunomolecular-magnetic beads for rapid detection and separation of S. aureus and group $G$ Streptococcus Preparation and characterization of polymer- coated core- shell structured magnetic microbeads[ J]. Applied Microbiology and Biotechnology, 2007, 75(5): 1209-1216.

[7] Dou. Yongzhong, Tomohiro T, Kodaka M. Synthesis of hemispherical poly (2-hydroxylethyl methacrylate-co-methyl methacrylate)/poly(styrene-co-glycidyl methacrylate) composite particles with heterobifunctional groups by soap-free seeded Emulsion polymerization[J]. Macromolecules, 2004, 37(3): 803-812.

[8] Y. Jian, Hu Fuqiang, Du Yongzhong. Polymeric micelles with glycolipid-like structure and multiple hydrophobic domains for mediating molecular targetdelivery of paclitaxel[J]. Biomacromolecules, 2007, 8(8): 2450-2456.

[9] Chang-jun zou. Spherical cellulose fixed Anti - HBsAg immune adsorbent monoclonal antibody preparation [J]. Chemical and biological engineering,2004(1): 32-33. 